\title{
DISTRIBUTION OF ZEROES TO GENERALIZED HERMITE POLYNOMIALS
}

\author{
DEDICATED TO 80 TH BIRTHDAY OF I.F. KRASICHKOV-TERNOVSKII
}

\section{V.YU. NOVOKSHENOV, A.A. SCHELKONOGOV}

\begin{abstract}
Asymptotics of the orthogonal polynomial constitute a classic analytic problem. In the paper, we find a distribution of zeroes to generalized Hermite polynomials $H_{m, n}(z)$ as $m=n, n \rightarrow \infty, z=O(\sqrt{n})$. These polynomials defined as the Wronskians of classic Hermite polynomials appear in a number of mathematical physics problems as well as in the theory of random matrices. Calculation of asymptotics is based on Riemann-Hilbert problem for Painlevé IV equation which has the solutions $u(z)=-2 z+\partial_{z} \ln H_{m, n+1}(z) / H_{m+1, n}(z)$. In this scaling limit the Riemann-Hilbert problem is solved in elementary functions. As a result, we come to analogues of Plancherel-Rotach formulae for asymptotics of classical Hermite polynomials.
\end{abstract}

Keywords. Generalized Hermite polynomials, Painlevé IV equation, meromorphic solutions, distribution of zeroes, Riemann-Hilbert problem, Deift-Zhou method, PlancherelRotach formulae.

Mathematics Subject Classification: $30 \mathrm{D} 35$, 30E10, 33C75, 34M35, 34M55, 34M60

\section{INTRODUCTION}

Generalized Hermite polynomials $H_{m, n}(z)$ are defined by the formulae [17], [4]

$$
H_{m, n}(z)=\operatorname{det}\left(P_{n-i+j}(z)\right)_{i, j=1}^{m},
$$

where

$$
P_{s}(z)=\sum_{i+2 j=s} \frac{1}{6^{j} i ! j !} z^{i}
$$

or, equivalently, as Wronskians of classical Hermite polynomials

$$
H_{m, n}(z)=c_{m, n} \mathcal{W}\left(H_{m}(z), H_{m+1}(z), \ldots, H_{m+n}(z)\right),
$$

where $H_{n}(z)=(-1)^{n} e^{z^{2}} \frac{d^{n}}{d z^{n}} e^{-z^{2}}, c_{m, n}$ are normalization constants.

Like classical orthogonal polynomials, they possess many remarkable properties. For instance, polynomials $p_{n}(x)$ orthogonal on the real axis with the weight $w(x, z, m)=(x-z)^{m} \exp \left(-x^{2}\right)$ satisfy the identity [3]

$$
x p_{n}(x)=p_{n+1}(x)+a_{n}(z, m) p_{n}(x)+b_{n}(z, m) p_{n-1}(x),
$$

V.Yu. Novokshenov, A.A. Schelkonogov, Distribution of zeroes to generalized Hermite POLYNOMIALS.

(c) Novokshenov V.Yu., Schelkonogov A.A. 2015.

The research is supported by the grant of Russian Scientific Foundation (project no. 14-11-00078).

Submitted August 24, 2015. 
where

$$
a_{n}(z, m)=-\frac{1}{2} \frac{d}{d z} \ln \frac{H_{n+1, m}}{H_{n, m}}, \quad b_{n}(z, m)=\frac{n H_{n+1, m} H_{n-1, m}}{2 H_{n, m}^{2}} .
$$

Another property which will be used in what follows is that the logarithmic derivatives

$$
u(z)=-2 z+\frac{d}{d z} \ln \frac{H_{m, n+1}(z)}{H_{m+1, n}(z)}
$$

are rational solutions to the fourth Painlevé equation (PIV)

$$
u^{\prime \prime}=\frac{\left(u^{\prime}\right)^{2}}{2 u}+\frac{3}{2} u^{3}+4 z u^{2}+2\left(z^{2}-\alpha\right) u+\frac{\beta}{2 u}
$$

with the coefficients

$$
\alpha=n-m, \quad \beta=-2(m+n+1)^{2} .
$$

We also mention a recently found application related to matrix models in the statistical physics. The distribution function for eigenvalues in Gaussian unitary ensemble [10] under fixing $n$ eigenvalues $\lambda_{k}$ and $m$-fold degeneration of $(n+1)$-th eigenvalue $\lambda_{n+1}=z$ reads as

$$
D_{n}(z)=\frac{1}{n !} \int_{\infty}^{\infty} \ldots \int_{\infty}^{\infty} \prod_{1 \leqslant i<j \leqslant n}\left(\lambda_{i}-\lambda_{j}\right)^{2} \prod_{k=1}^{n}\left(\lambda_{k}-z\right)^{m} e^{-\lambda_{k}^{2}} d \lambda_{k} .
$$

On other hand, statistical sum (4) can be considered as the energy of the ground state of the wave function for $n+1$ Coulomb particles with $1 / r^{2}$ repulsion in an external quadratic field [10]. At that, it happens [3] that

$$
D_{n}(z)=A_{m, n} H_{m, n}(c z), \quad c=i \sqrt{\frac{2}{3}}, \quad A_{m, n}=\text { const. }
$$

Thus, the zeroes of generalized Hermite polynomials correspond to the coordinates of Coulomb particles in equilibrium state hold by the external quadratic field.

The calculation of asymptotic distribution of orthogonal polynomials go back to the works of classics in analysis in the end of XIX century, like Darboux, Chebyshev, Steklov and Stiltjes. In particular, for Hermite polynomials, this distribution is implied by Plancherel-Rotach formulae, [14, [16, Ch. 8],

$$
e^{-z^{2} / 2} H_{n}(z)=\frac{2^{\frac{n}{2}+\frac{1}{4}} \sqrt{n !}}{(\pi n)^{1 / 4} \sqrt{\sin \varphi}}\left\{\sin \left[\left(\frac{n}{2}+\frac{1}{4}\right)(\sin 2 \varphi-2 \varphi)+\frac{3 \pi}{4}\right]+O\left(n^{-1}\right)\right\},
$$

where $z=\sqrt{2 n+1} \cos \varphi, \varepsilon \leqslant \varphi \leqslant \pi-\varepsilon, n \rightarrow \infty$ and

$$
e^{-z^{2} / 2} H_{n}(z)=\frac{2^{\frac{n}{2}-\frac{3}{4}} \sqrt{n !}}{(\pi n)^{1 / 4} \sqrt{\operatorname{sh} \varphi}}\left\{\exp \left[\left(\frac{n}{2}+\frac{1}{4}\right)(\operatorname{sh} 2 \varphi-2 \varphi)+\frac{3 \pi}{4}\right]+O\left(n^{-1}\right)\right\},
$$

where $z=\sqrt{2 n+1} \operatorname{ch} \varphi, \varepsilon \leqslant \varphi \leqslant \omega, n \rightarrow \infty, \omega$ is an arbitrary constant.

It follows that zeroes of $H_{n}(z)$ are real and are located in the domain of the characteristic scale $O(\sqrt{2 n})$.

In what follows we calculate the distribution of zeroes of polynomials $H_{m, n}(z)$ as

$$
m=n, \quad n \rightarrow \infty, \quad z=O(\sqrt{n}) .
$$

In order to do it, after the example of recent works [1, [2] and [13, we employ rational solutions (2) to equation (3). It is easy to show that the poles of this solution with residues +1 correspond to the zeroes of polynomials $H_{m, n+1}$, with the poles with residues -1 coincide with the zeroes of polynomial $H_{m+1, n}$. Then, using the complete integrability of PIV equation, we can formulate Riemann-Hilbert problem corresponding to given rational solutions. In order 
FiguRE 1. Zeroes of generalized Hermite polynomials $H_{m, n}(z)$ for $m=n=10$ (left) and for $m=15$ and $n=10$ (right)

to do it, in Section 1 we calculate monodromy data and a chain of Bäcklund transformation leading to solutions (2). This matrix problem happens to be very similar to the corresponding Riemann-Hilbert problem applied for the family of orthogonal polynomials with an exponential weight (Section 2). The method of asymptotic solving such problems consists in providing a special parametrix regularizing a power growth at infinity with replacing it by a standard normalization by the unit matrix. In the present case, this method going back to works by $\mathrm{P}$. Deift and others [5], is similar to a procedure serving classical Hermite polynomials. We show in Section 3 that the parametrix is constructed in terms of elementary functions as opposed to the case of Vorobiev-Yablonski polynomials in PII equation leading to the parametrix in terms of theta functions [1], 2]. As a result, in Section 4 we recover the lattice of zeroes of generalized Hermite polynomials in the limit (5) be resolving a system of trigonometric equations.

\section{BÄCKLUND TRANSFORMATION AND CALCULATION OF MONODROMY DATA}

The first integrals of PIV equation are monodromy data defined as follows [8, Ch. 5]. We consider a linear matrix equation w.r.t. an additional parameter $\lambda$

$$
\Psi_{\lambda}=A(\lambda, z) \Psi
$$

where $\Psi=\Psi(\lambda, z)$ is $2 \times 2$-matrix function, and $A(\lambda, z)$ is rational in $\lambda$

$$
\begin{aligned}
A= & \lambda\left(\begin{array}{cc}
1 & 0 \\
0 & -1
\end{array}\right)+\left(\begin{array}{cc}
z & y \\
2\left(u w+\theta_{0}-\theta_{\infty}\right) y^{-1} & -z
\end{array}\right) \\
& +\frac{1}{\lambda}\left(\begin{array}{cc}
-u w+\theta_{0} & -y w / 2 \\
2\left(u w-2 \theta_{0}\right) y^{-1} & u w-\theta_{0}
\end{array}\right) .
\end{aligned}
$$

Here $u, w$ and $y$ functions of $z$ satisfying nonlinear equations

$$
\begin{gathered}
\frac{d}{d z} \log y=-u-2 z, \\
\left\{\begin{array}{l}
\frac{d u}{d z}=-4 u w+u^{2}+2 z u+4 \theta_{0}, \\
\frac{d w}{d z}=2 w^{2}-2 u w-2 z w+\theta_{0}+\theta_{\infty} .
\end{array}\right.
\end{gathered}
$$

Equations (7) are equivalent to PIV equation (3) for function $u=u(z)$ with the coefficients

$$
\alpha=2 \theta_{\infty}-1, \quad \beta=-8 \theta_{0}^{2} .
$$


The monodromy data are defined by Stokes matrix for solutions $\Psi$ to equation (6)

$$
\Psi_{j+1}(\lambda, z)=\Psi_{j}(\lambda, z) S_{j}, \quad j=1,2,3,4 .
$$

Here four fundamental solutions $\Psi_{j}$ to equation (6) are chosen by the condition of analyticity in $\lambda$ in sector $\Omega_{j}$,

$$
\Omega_{j}=\{\lambda \mid-\pi / 4+\pi j / 2<\arg \lambda<\pi / 4+\pi j / 2, \quad j=1,2,3,4\},
$$

and the normalization at infinity

$$
\begin{aligned}
& \Psi_{j}(\lambda, z)=\left(I+O\left(\lambda^{-1}\right)\right) \exp \left\{\left(\frac{\lambda^{2}}{2}+z \lambda\right) \sigma_{3}\right\} \lambda^{-\theta_{\infty} \sigma_{3}}, \\
& \lambda \rightarrow \infty, \quad \lambda \in \Omega_{j}, \quad \sigma_{3}=\left(\begin{array}{cc}
1 & 0 \\
0 & -1
\end{array}\right) .
\end{aligned}
$$

The main feature of Stokes matrices $S_{j}(8)$ is that they are independent of $z$ if and only if $u, w$, and $y$ satisfy system (7) or, equivalently, $u=u(z)$ solves PIV equation (3) ([8], [11]). Thus, scalar entries of these matrices $s_{1}, s_{2}, s_{3}$ and $s_{4}$

$$
S_{2 k}=\left(\begin{array}{cc}
1 & s_{2 k} \\
0 & 1
\end{array}\right), \quad S_{2 k-1}=\left(\begin{array}{cc}
1 & 0 \\
s_{2 k-1} & 1
\end{array}\right), \quad k=1,2
$$

become motion integrals (first integrals) of PIV equation (3). It is obvious that they are independent since a second order equation has just two independent first integrals. Indeed, the monodromy data satisfy the relation [8, Ch. 5],

$$
\left(1+s_{2} s_{3}\right) e^{2 \pi i \theta_{\infty}}+\left(s_{1} s_{4}+\left(1+s_{3} s_{4}\right)\left(1+s_{1} s_{2}\right)\right) e^{2 \pi i \theta_{\infty}}=2 \cos \left(2 \pi \theta_{0}\right)
$$

implied by the cyclicity condition in the iteration of monodromy equations (8)

$$
S_{1} S_{2} S_{3} S_{4} e^{2 \pi i \theta_{\infty} \sigma_{3}}=T e^{2 \pi i \theta_{0} \sigma_{3}} T^{-1}, \quad \operatorname{det} T=1 .
$$

The inverse problem of monodromy theory for equation PIV is to find $\Psi_{j}$ satisfying conditions (8) and (9) with given monodromy data $s_{1}, s_{2}, s_{3}, s_{4}$ under condition (10). If such functions exist, the solution to PIV equation (3) is found by formula ([8, Ch. 5])

$$
u(z)=-2 z-\lim _{\lambda \rightarrow \infty}\left(\lambda \partial_{z} \Psi_{1}(\lambda, z) \exp \left\{-\left(\frac{\lambda^{2}}{2}+z \lambda\right) \sigma_{3}\right\} \lambda^{-\theta_{\infty} \sigma_{3}}\right)_{12},
$$

where the subscript ${ }_{12}$ denotes the element $(1,2)$ of matrix $\Psi_{1}$.

Proceeding to solving this problem as $z \rightarrow \infty$, we first calculate $s_{j}$ for rational solutions (2). In order to od it, we employ recurrent relations for generalized Hermite polynomials. All $H_{m, n}$ are related by the identities [4]

$$
\left\{\begin{array}{l}
2 m H_{m+1, n} H_{m-1, n}=H_{m, n} H_{m, n}^{\prime \prime}-\left(H_{m, n}^{\prime}\right)^{2}+2 m H_{m, n}^{2} \\
2 n H_{m, n+1} H_{m, n-1}=-H_{m, n} H_{m, n}^{\prime \prime}+\left(H_{m, n}^{\prime}\right)^{2}+2 n H_{m, n}^{2}
\end{array}\right.
$$

with "initial conditions"

$$
H_{0,0}=H_{0,1}=H_{1,0}=1, \quad H_{1,1}=2 z .
$$

The correpoding solutions to PIV equations (2) a

$$
u_{m, n}(z)=-2 z+\frac{d}{d z} \ln \frac{H_{m, n+1}(z)}{H_{m+1, n}(z)}
$$

also satisfy recurrences called Bäcklund transformations. These are nonlinear transformations $\widetilde{u}(z)=\mathcal{R}\left(u^{\prime}(z), u(z), z\right)$ mapping solution $u(z)$ into solution $\tilde{u}(z)$ of PIV equation with possible other coefficients $\alpha, \beta$. 
We consider a pair of Bäcklund transformations [4]

$$
\begin{aligned}
& \mathcal{R}_{2}: \quad \tilde{u}=\frac{\left(u^{\prime}-\sqrt{-2 \beta}\right)^{2}+(4 \alpha-4-2 \sqrt{-2 \beta}) u^{2}-u^{2}(u+2 z)^{2}}{2 u\left(u^{2}+2 z u+u^{\prime}-\sqrt{-2 \beta}\right)}, \\
& \mathcal{R}_{3}: \quad \tilde{u}=\frac{\left(u^{\prime}-\sqrt{-2 \beta}\right)^{2}-(4 \alpha+4+2 \sqrt{-2 \beta}) u^{2}-u^{2}(u+2 z)^{2}}{2 u\left(u^{2}+2 z u-u^{\prime}+\sqrt{-2 \beta}\right)} .
\end{aligned}
$$

The composition of transformations $\mathcal{R}_{6}=\mathcal{R}_{2} \mathcal{R}_{3}$ happens to map the coefficients $(\alpha, \beta)$ into $\left(\alpha,-\frac{1}{2}(4+\sqrt{-2 \beta})^{2}\right)$, and parameters $\left(\theta_{0}, \theta_{\infty}\right)$ are mapped into $\left(\theta_{0}+2, \theta_{\infty}\right)$, respectively. It is easy to check that for rational solutions (14) with coefficients $\alpha=n-m, \beta=-2(m+n+1)^{2}$ we finally get the solutions

$$
\tilde{u}(z)=\mathcal{R}_{6}\left(u_{m, n}^{\prime}(z), u_{m, n}(z), z\right)=u_{m+1, n+1}(z) .
$$

In terms of $\Psi$-functions and "equation in $\lambda$ " (6), Bäcklund transformation correspond to the left multiplication by matrices rational in $\sqrt{\lambda}$ ("dressing" of Lax pair [8, Ch. 6])

$$
\tilde{\Psi}=\mathbf{R}_{6}(\lambda) \Psi \text {. }
$$

For transformations (15) these matrices read as

$$
\begin{aligned}
& \mathbf{R}_{2}(\lambda)=\left(\begin{array}{ll}
1 & 0 \\
0 & 0
\end{array}\right) \lambda^{1 / 2}+\left(\begin{array}{cc}
y & \frac{y}{2} \\
\frac{2 y}{u} & 1
\end{array}\right) \lambda^{-1 / 2} \\
& \mathbf{R}_{3}(\lambda)=\left(\begin{array}{ll}
0 & 0 \\
0 & 1
\end{array}\right) \lambda^{1 / 2}+\left(\begin{array}{cc}
1 & \frac{u}{2 y} \\
-\frac{y w-\theta_{0}-\theta_{\infty}}{y} & -\frac{w y-\theta_{0}-\theta_{\infty}}{2 y}
\end{array}\right) \lambda^{-1 / 2}, \\
& \mathbf{R}_{6}(\lambda)=\mathbf{R}_{2}(\lambda) \mathbf{R}_{3}(\lambda)
\end{aligned}
$$

where quantities $y, u$ and $w$ are constant in $\lambda$ and satisfy system (7). The straightforward checking by means of "equation in $\lambda$ " (6) shows the equivalence of (16) and (15).

It is sufficient to calculate $s_{j}$ for the case $(13)$. We choose $m=n=0$, then by equations (14) and (13) we fins $u_{0,0}(z)=u(z)=-2 z$. Resolving other equations (7), we have

$$
y(z)=w(z)=1, \quad u(z)=-2 z .
$$

Substituting quantities (17) and $z=0$ into "equation in $\lambda$ " (6), we obtain a triangular matrix

$$
A=\left(\begin{array}{cc}
\lambda+\frac{1}{2 \lambda} & 1 \\
0 & -\lambda-\frac{1}{2 \lambda}
\end{array}\right) .
$$

Hence, equation (6) is solved explicitly

$$
\Psi_{j}(\lambda, 0)=\left(\begin{array}{cc}
\lambda^{1 / 2} e^{\frac{\lambda^{2}}{2}} & \lambda^{1 / 2} e^{\frac{\lambda^{2}}{2}} \int_{\lambda}^{\infty} \frac{e^{\frac{-\xi^{2}}{2}}}{\xi} d \xi \\
0 & \lambda^{-1 / 2} e^{\frac{-\lambda^{2}}{2}}
\end{array}\right), \quad \lambda \in \Omega_{j},
$$

and the integration is made over the rays $\arg \lambda=\frac{\pi}{4}+\frac{\pi}{2} j, j=1,2,3,4$.

Theorem 1. Monodromy data $s_{j}$ for "equation in $\lambda$ " (6) with coefficients (17) corresponding to the solution $u(z)=u_{0,0}=-2 z$ read as

$$
s_{1}=s_{3}=0, \quad s_{2}=2 \pi i, \quad s_{4}=-2 \pi i .
$$


Proof. Since matrices $\Psi_{j}(18)$ are upper-triangular, jump equation (8) has also upper-triangular solutions. It yields $S_{1}=S_{3}=I$ so that for odd $j=1,3$ the monodromy data is zero. To calculate $S_{2}$ and $S_{4}$, we observe that

$$
\Psi_{3}=\Psi_{2} S_{2}=\Psi_{1} S_{1} S_{2}=\Psi_{1} S_{2} .
$$

Then

$$
s_{2}=\left(\Psi_{3} \Psi_{1}^{-1}\right)_{12}=\int_{\infty e^{5 \pi / 4 i}}^{\infty e^{\pi / 4 i}} \frac{e^{-\frac{\xi^{2}}{2}}}{\xi} d \xi
$$

where the integration contour passes the origin from above. Thus, the contour for the integral exponent function in this formula is deformed into the passage of the origin in the negative direction. It gives $s_{2}=-2 \pi i$. In the same way we calculate $s_{4}$.

Let us formulate Riemann-Hilbert problem which will be employed below for calculating the solutions to PIV equation (14). We observe first that by Theorem $11 \Psi_{1}=\Psi_{2}$ and $\Psi_{3}=\Psi_{4}$ since there are no jumps on the rays $\arg \lambda=3 \pi / 4$ and $\arg \lambda=7 \pi / 4$. Moreover, conjugation condition (8) can be moved from the rays $\arg \lambda=\pi / 4$ and $\arg \lambda=5 \pi / 4$ on the real axis that does not contradict to normalization (9).

First we make the change of variables taking into consideration large parameters $z=O(\sqrt{n})$, $n=m \rightarrow \infty$ (5)

$$
z=x \sqrt{n}, \quad \lambda=(\xi-x) \sqrt{n}, \quad \Theta=\frac{1}{2}\left(\xi^{2}-x^{2}\right) .
$$

We introduce new matrix functions

$$
\begin{aligned}
& Y_{+}(\lambda, z)=\Psi_{4}(\lambda, z) \exp \left\{-\left(\frac{\lambda^{2}}{2}+z \lambda\right) \sigma_{3}\right\} \lambda^{-\theta_{\infty} \sigma_{3}}, \\
& Y_{-}(\lambda, z)=\Psi_{2}(\lambda, z) \exp \left\{-\left(\frac{\lambda^{2}}{2}+z \lambda\right) \sigma_{3}\right\} \lambda^{-\theta_{\infty} \sigma_{3}} .
\end{aligned}
$$

Functions $Y_{+}$and $Y_{-}$satisfy the following conditions

1 Matrix function $Y_{+}(\xi, x)$ is analytic in $\xi$ in the upper half-plane $\operatorname{Im} \xi>0$, and $Y_{-}(\xi, x)$ is analytic in the lower half-plane $\operatorname{Im} \xi<0$

2

$$
Y_{+}(\xi, x)=Y_{-}(\xi, x)\left(\begin{array}{cc}
1 & 2 \pi i e^{-2 n \Theta(\xi, x)} \\
0 & 1
\end{array}\right), \quad \xi \in \mathbb{R}
$$

3

$$
Y_{ \pm}(\xi, x)=\left(I+O\left(\xi^{-1}\right)\right)\left(\begin{array}{cc}
\xi^{2 n} & 0 \\
0 & \xi^{-2 n}
\end{array}\right), \quad \xi \rightarrow \infty
$$

Here we have shifted the contour of conjugation $\mathbb{R} \mapsto \mathbb{R}+x$ since the conjugation matrix is analytic in the strip $|\operatorname{Im} \xi| \leqslant|x|, x=O(1)$. We also observe that normalization condition 3 appears due to $n$-multiple application of Bäcklund transformation (16) to matrices $\Psi_{j}(18)$. Indeed, by the structure of matrices $\mathbf{R}_{2}(\lambda)$ and $\mathbf{R}_{3}(\lambda)$, their product multplies $(\Psi)_{11}$ by $\lambda$ and $(\Psi)_{22}$ by $\lambda^{-1}$.

Now the inversion formula for solutions to PIV equation becomes simpler.

Theorem 2. Suppose that matrices $Y_{ \pm}$solve Riemann-Hilbert problem (21). Then the function

$$
u_{n, n}(z)=-2 x \sqrt{n}-\lim _{\xi \rightarrow \infty}\left(\xi \partial_{x} Y_{+}(\xi, x)\right)_{12}, \quad z=x \sqrt{n}
$$

solve PIV equation (3) corresponding to monodromy data (19). 
Proof. By matrices $Y_{ \pm}$we recover matrices $\Psi_{j}$ satisfying conditions (8) and (9) with monodromy data (19). Then "equation in $\lambda$ " (6) holds true and inversion formula (11) is valid; the latter formula coincides with 22 .

\section{ORTHOGONAL POLYNOMIALS WITH EXPONENTIAL WEIGHT}

Before proceeding to asymptotic study of Riemann-Hilbert problem (21), let us show its connection with orthogonal polynomials. Let $\left\{\mathcal{H}_{k}(\xi)\right\}_{k=1}^{\infty}$ be the set of polynomials $\mathcal{H}_{k}(\xi)=$ $\xi^{k}+\ldots$ orthogonal on the real axis with the measure $e^{-n V(\xi)} d \xi$

$$
\int_{\mathbb{R}} \mathcal{H}_{j}(\xi) \mathcal{H}_{k}(\xi) e^{-n V(\xi)} d \xi=0, \quad j \neq k
$$

where $V(\xi)=\xi^{2 l}+\ldots$ is a polynomial of even degree. We define the matrices

$$
Y^{(q)}(\xi)=\left(\begin{array}{cc}
\mathcal{H}_{q}(\xi) & \frac{1}{2 \pi i} \int_{\mathbb{R}} \frac{\mathcal{H}_{q}(s) e^{-n V(s)}}{s-\xi} d s \\
\gamma_{q-1} \mathcal{H}_{q-1}(\xi) & \frac{\gamma_{q-1}}{2 \pi i} \int_{\mathbb{R}} \frac{\mathcal{H}_{q-1}(s) e^{-n V(s)}}{s-\xi} d s
\end{array}\right), \quad \operatorname{Im} \xi \neq 0
$$

where constants $\gamma_{q-1}$ are the normalization ones in the orthogonality condition

$$
\gamma_{q-1}=-2 \pi i\left(\int_{\mathbb{R}} \mathcal{H}_{q-1}^{2}(s) e^{-n V(s)} d s\right)^{-1} .
$$

Matrices (23) happen to solve the following Riemann-Hilbert problem ([9], [5, Ch. 3.2])

- $Y_{+}^{(q)}(\xi)$ analytic as $\operatorname{Im} \xi>0$, and $Y_{-}^{(q)}(\xi)$ analytic as $\operatorname{Im} \xi<0$

- $Y_{+}^{(q)}(\xi)=Y_{-}^{(q)}(\xi)\left(\begin{array}{cc}1 & e^{-n V(\xi)} \\ 0 & 1\end{array}\right), \quad \xi \in \mathbb{R}$

- $Y_{ \pm}^{(q)}(\xi, x)=\left(I+O\left(\xi^{-1}\right)\right)\left(\begin{array}{cc}\xi^{q} & 0 \\ 0 & \xi^{-q}\end{array}\right), \quad \xi \rightarrow \infty$

In the case $V(\xi)=\xi^{2}$ polynomials $\mathcal{H}_{q}(\xi)$ coincide with Hermite polynomials $H_{q}(\xi)=$ $(-1)^{q} e^{\xi^{2}} \frac{d^{q}}{d \xi^{q}} e^{-\xi^{2}}$ and the Riemann-Hilbert problem can be employed for calculating the asymptotics of Hermite polynomials as $q=n, n \rightarrow \infty$ [5, Sect. 7.4]. At that, normalization constants $\gamma_{q}$ read as

$$
\gamma_{q-1}=\left(Y_{1}^{(q)}\right)_{21}=-i \frac{2^{q} \sqrt{\pi} n^{(q-1) / 2}}{(q-1) !}
$$

where $Y^{(q)}(\xi)=\left(I+Y_{1}^{(q)} \xi^{-1}+\ldots\right) \xi^{(q-1) \sigma_{3}}$.

In the same way one can reproduce Plancherel-Rotach formulae mentioned in Introduction [5], [12]. Since Hermite polynomials have zeroes only in the real axis, the rows of matrix $Y^{(n)}$ do not vanish in the upper (lower) half-plane, i.e., the matrices are everywhere non-degenerate and Riemann-Hilbert problem is uniquely solvable.

The situation becomes much more complicated once the exponential weight becomes depending on a parameter. Indeed, replacing $V(\xi)$ by $\Theta(\xi, x)=\xi^{2}+2 \xi x$, let us consider Cauchy integrals in the second column of matrix $Y^{(n)}$

$$
\frac{1}{2 \pi i} \int_{\mathbb{R}} \frac{\mathcal{H}_{n}(s, x) e^{-n\left(s^{2}+2 s x\right)}}{s-\xi} d s=\frac{\mathcal{H}_{n}(-x, x) e^{-n x^{2}}}{2 i \pi^{5 / 4} \sqrt{2 x n}(-x-\xi)}\left(1+O\left(n^{-1}\right)\right), \quad n \rightarrow \infty .
$$


Here we have employed the saddle point method having deformed integration contour to make it passing through the point $s=-x, \operatorname{Im} x \neq 0$ so that the saddle point condition $\partial_{s}\left(s^{2}+2 s x\right)=0$ is satisfied. Let $\mathcal{H}_{n}(-x, x)=0$, then the first row of matrix (23) vanishes as $\xi=-x$. Thus, matrices $Y_{ \pm}^{(n)}$ become degenerate at this point and do not solve the Riemann-Hilbert problem.

The described situation is the implication of Birkhoff-Grothendieck theorem on factorization of a matrix holomorphically depending on a parameter [8, App. B].

\section{Asymptotic "UndREssing" of Riemann-Hilbert PROBlem}

In this section we find solution to Riemann-Hilbert problem (21) as $n \rightarrow \infty$. Despite this Riemann-Hilbert problem involves upper-triangular matrices only, its solution is not reduced to a sequence of scalar conjugate problems for analytic functions. The reason is normalization condition 3. Indeed, we can get rid of the power growth at infinity by introducing the matrices $\tilde{Y}_{ \pm}=Y_{ \pm} \operatorname{diag}\left(z^{-2 n}, z^{2 n}\right)$. Then matrices $\tilde{Y}_{ \pm}$get the standard normalization by the unit matrix $\tilde{Y}_{ \pm} \rightarrow I$ as $\xi \rightarrow \infty$. However, a pole on $2 n$-th order appear at zero. This fact prevents applying of Cauchy integral to conjugation problem (21).

To regularize matrices $Y_{ \pm}$at infinity, we employ another approach created by Percy Deift and others in [5], [6], [7]. It consists in applying a special parametrix called $g$-function. In our case it is an analytic function satisfying the conditions

- $g(\xi)=\int_{-a}^{a} \ln (\xi-s) \rho(s) d s, \quad a \in \mathbb{R}, \quad a=O(1)$

- $g_{ \pm}(\xi)=\int_{-a}^{a} \ln |\xi-s| \rho(s) d s \pm \pi i \chi_{\xi \leqslant a} \int_{\xi}^{a} \rho(s) d s$ as $\xi \in(-a, a), \chi_{\xi \leqslant a}$ is the characteristic function of $[\xi, a]$

- $g(\xi)=\ln \xi+O\left(\xi^{-1}\right), \quad \xi \rightarrow \infty$

- $e^{n g(\xi)}$ is analytic in $\mathbb{C} \backslash[-a, a]$

- $e^{n g(\xi)}=\xi^{n}\left(1+O\left(\xi^{-1}\right)\right), \quad \xi \rightarrow \infty$

Function $\rho(s)$ is interpreted as the probability measure $d \mu=\rho(s) d s$ minimizing the functional

$$
\mathcal{F}=\int_{\mathbb{R}} \xi^{2} d \mu(\xi)+\int_{\mathbb{R}^{2}} \ln |\xi-s|^{-1} d \mu(\xi) d \mu(s), \quad x=\text { const. }
$$

Theorem 3. [15, Ch. 5] As $\partial_{\xi}^{2} \Theta(\xi, x) \geqslant 0$, the support of measure $d \mu=\rho(s) d s$ is located in the segment $\operatorname{supp} \rho \in[-a, a]$. The relations

$$
\begin{aligned}
& 2 \Theta(\xi, x)+2 \int_{\mathbb{R}} \ln |\xi-s|^{-1} \rho(s) d s+\ell+x^{2} \geqslant 0, \quad \xi \in \mathbb{R}, \\
& 2 \Theta(\xi, x)+2 \int_{\mathbb{R}} \ln |\xi-s|^{-1} \rho(s) d s+\ell x^{2} \equiv 0, \quad \xi \in[-a, a],
\end{aligned}
$$

hold true, where

$$
\ell=-2 \int_{-a}^{a} \ln |\xi-s|^{-1} \rho(s) d s-\xi^{2}, \quad-a<\xi<a
$$


Define the function

$$
\varphi(\xi) \equiv \xi^{2}-2 g(\xi)+\ell
$$

and a new piece-wise analytic matrix

$$
W(\xi, x) \equiv e^{n \ell \sigma_{3}} Y(\xi, x) e^{-n g(\xi) \sigma_{3}} e^{-n \ell \sigma_{3}}, \quad \xi \in \mathbb{C} / \mathbb{R} .
$$

Then matrix $W$ solves the following Riemann-Hilbert problem

- $W(\xi, x)$ is analytic w.r.t. $\xi$ in $\mathbb{C} \backslash \mathbb{R}$

$\begin{array}{ll}\text { - } W_{+}(\xi, x)=W_{+}(\xi, x)\left(\begin{array}{cc}e^{n\left(\varphi_{+}-\varphi_{-}\right)} & e^{-n\left(\varphi_{+}+\varphi_{-}\right)} \\ 0 & e^{-n\left(\varphi_{+}-\varphi_{-}\right)}\end{array}\right), \quad \xi \in \mathbb{R} & W(\xi, x) \\ \text { - } W_{ \pm}(\xi, x)=I+O\left(\xi^{-1}\right), \text { as } \xi \rightarrow \infty & \end{array}$

Thus, the problem is reduced to the Riemann-Hilbert problem with the standard unit normalization at infinity. As usually, here $\varphi_{+}$and $\varphi_{-}$are the boundary values on the real axis from the upper and lower half-plane, respectively. Let us list the properties of these boundary values as a theorem by P. Deift.

Theorem 4. [5, Ch. 7.5] Boundary values of function $\varphi$ (27) have the properties:

1) $\frac{-\varphi_{+}+\varphi_{-}}{2}=g_{+}(\xi)-g_{-}(\xi)=2 \pi i \int_{\xi}^{\infty} \rho(s) d s$ as $\xi \in \mathbb{R}$

2) $-\frac{1}{2} \operatorname{Im} \varphi_{+}=\operatorname{Im} g_{+}(\xi)=\pi \int_{-\infty}^{\xi} \rho(s) d s-\pi$ as $\xi \in[-a, a]$

3) $\frac{\varphi_{+}+\varphi_{-}}{2}=\operatorname{Re} \varphi=\xi^{2}-g_{+}-g_{-}+\ell \equiv 0$ as $\xi \in[-a, a]$

4) $\frac{\varphi_{+}+\varphi_{-}}{2}=\operatorname{Re} \varphi=\xi^{2}-g_{+}-g_{-}+\ell \geqslant 0$ as $\xi \notin[-a, a]$

We note that property 2 means that $\operatorname{Re} \varphi_{+}$decays as $\operatorname{Im} \xi \rightarrow+\infty$ by Cauchy-Riemann equation $u_{y}=-v_{x}, \varphi=u+i v$. In the same way, $\operatorname{Re} \varphi_{-}$decays as $\operatorname{Im} \xi \rightarrow-\infty$ since $\operatorname{Im} \varphi_{-}=$ $-\operatorname{Im} \varphi_{+}$.

Conjugation matrix involved in $(29)$ can be factorized [5, Ch. 7.6]:

$$
\left(\begin{array}{cc}
e^{n\left(\varphi_{+}-\varphi_{-}\right)} & e^{-n\left(\varphi_{+}+\varphi_{-}\right)} \\
0 & e^{-n\left(\varphi_{+}-\varphi_{-}\right)}
\end{array}\right)=\left(\begin{array}{cc}
1 & 0 \\
e^{2 n \varphi_{-}} & 1
\end{array}\right)\left(\begin{array}{cc}
0 & e^{-n\left(\varphi_{+}+\varphi_{-}\right)} \\
-e^{n\left(\varphi_{+}+\varphi_{-}\right)} & 0
\end{array}\right)\left(\begin{array}{cc}
1 & 0 \\
e^{2 n \varphi_{+}} & 1
\end{array}\right) .
$$

Thus, problem $(29)$ can be rewritten as

$$
\begin{aligned}
& \Phi_{+}=\Phi_{-}\left(\begin{array}{cc}
0 & e^{-n\left(\varphi_{+}+\varphi_{-}\right)} \\
-e^{n\left(\varphi_{+}+\varphi_{-}\right)} & 0
\end{array}\right), \quad \xi \in \mathbb{R}, \\
& \Phi_{ \pm}(\xi, x) \rightarrow I, \quad \xi \rightarrow \infty
\end{aligned}
$$

where

$$
\Phi_{ \pm}(\xi, x) \equiv W_{ \pm}(\xi, x)\left(\begin{array}{cc}
1 & 0 \\
\mp e^{2 n \varphi} & 1
\end{array}\right), \quad \operatorname{Im} \xi \gtrless 0 .
$$

By Theorem 4, $\varphi_{+}+\varphi_{-} \equiv 0$ as $\xi \in[-a, a]$, while $\varphi_{+}+\varphi_{-}$is non-negative for $\xi \in \mathbb{R} \backslash[-a, a]$. This is why Riemann-Hilbert problem for matrices $\Phi$ and $W$ is introduced as it is shown in Fig. 2. It is easy to check that all the conjugation matrices beyond the "lens" $\operatorname{Re} \varphi<0$ marked by the thick line are exponentially close to the unit one since their off-diagonal elements are of order $O\left(e^{-n|\varphi|}\right)$. The neighbourhoods of boundary points $\xi=a$ and $\xi=-a$ deserve a separate 
FiguRE 2. Regularization of Riemann-Hilbert problem (21) by Deift-Zhou. The jump matrices are associated with Stokes lines.

consideration. However, it happens that the values of $\Phi$ can be analytically continued into solutions $\Phi$ for the interior of the "lens" [5, Ch. 7.6].

Thus, the leading term in $n$, the solution to Riemann-Hilbert problem (31) is provided by matrix $\Phi$ satisfying the model problem

$$
\begin{aligned}
& \Phi_{+}=\Phi_{-}\left(\begin{array}{cc}
0 & 1 \\
-1 & 0
\end{array}\right), \quad \xi \in[-a, a], \\
& \Phi_{ \pm}(\xi) \rightarrow I, \quad \xi \rightarrow \infty .
\end{aligned}
$$

The solution to problem 32 is given by explicit formula [6]

$$
\Phi(\xi)=\frac{1}{2}\left(\begin{array}{ll}
1 & -i \\
i & -1
\end{array}\right)\left(\frac{\xi-a}{\xi+a}\right)^{\frac{1}{4} \sigma_{3}}\left(\begin{array}{ll}
1 & -i \\
i & -1
\end{array}\right) .
$$

To find the leading term of the asymptotics for $Y(\lambda, z)$, it remains to calculated quantities $g(\xi), \rho(s)$ and $a$ involved in problems (25), (29) and Theorems 3), 4. These quantities are also given by explicit formula via the approach of [5, Sect. 7.3]. Omitting details, we provide the result:

$$
\begin{aligned}
\rho(\xi) & =\frac{1}{\pi} \sqrt{a^{2}-\xi^{2}}+\frac{x}{\pi} \sqrt{\frac{a-\xi}{a+\xi}}, \\
g(\xi) & =-\ln \left(\xi-\sqrt{\xi^{2}-a^{2}}\right)+\frac{1}{4}\left(\xi-\sqrt{\xi^{2}-a^{2}}\right)^{2}, \\
a & =\sqrt{2} .
\end{aligned}
$$

Calculation of quantities $\rho(\xi), g(\xi)$ and $a$ are based on the explicit solution to scalar RiemannHilbert problem (25) and on the inversion formula implied by (22). Indeed, the assumptions of problem (25) can be easily checked by explicit formulae (34) and (35).

Combining the formulae obtained above in this section, asymptotics for the solution to Riemann-Hilbert problems (29), (31) and (32), we arrive at the following theorem.

Theorem 5. ([6]) The solution to Riemann-Hilbert problem (21) has the following asymptotics 
1) Outside the "lens" $\operatorname{Re} \varphi<0$ :

$$
Y(\lambda, z)=e^{n \ell \sigma_{3}} \Phi(\xi) e^{n(2 g-\ell) \sigma_{3}}\left(I+O\left(n^{-1}\right), \quad n \rightarrow \infty,\right.
$$

2) Inside the "lens" $\operatorname{Re} \varphi>0$ :

$$
Y(\lambda, z)=e^{n \ell \sigma_{3}} \Phi(\xi)\left(\begin{array}{cc}
1 & 0 \\
e^{2 n \varphi} & 1
\end{array}\right) e^{n(2 g-\ell) \sigma_{3}}\left(I+O\left(n^{-1}\right), \quad n \rightarrow \infty,\right.
$$

where $\Phi(\xi)$ is defined by formula (33), and $\varphi$ and $\ell$ are introduced by formulae (26) and (27).

\section{INVERSION FORMULA AND ASYMPTOTICS FOR ZEROES}

The leading term of the asymptotics for the solution to Riemann-Hilbert problem given in Theorem 5 allows us to calculate the asymptotics for polynomials $H_{n, n}(\lambda)$ with the exponential weight corresponding to this problem. We begin with case 1 ), where $|\xi|>\sqrt{2}$, i.e., $|z|>\sqrt{2 n}$. In accordance with explicit representation (23) for matrix $Y$ and expression (35) for function $g$ we have

$$
H_{n, n}(z)=(Y)_{11}(z, x)=\Phi_{11}(\xi) e^{2 n g(\xi)}\left(I+O\left(n^{-1}\right), \quad \xi=x, \quad z=x \sqrt{n},\right.
$$

that gives finally as $n \rightarrow \infty$ and $|x|>\sqrt{2}$

$$
H_{n, n}(x \sqrt{n}) \approx \frac{1}{2}\left[\left(\frac{x-\sqrt{2}}{x+\sqrt{2}}\right)^{\frac{1}{4}}+\left(\frac{x+\sqrt{2}}{x-\sqrt{2}}\right)^{\frac{1}{4}}\right] \frac{e^{\frac{n}{4}\left(x-\sqrt{x^{2}-2}\right)^{2}}}{\left(x-\sqrt{x^{2}-2}\right)^{n}} .
$$

In case 2) as $|\xi|<\sqrt{2}$, the additional matrix factor in Theorem 5 gives

$$
\begin{aligned}
& H_{n, n}(z)=(Y)_{11}(z, x)=\left(\Phi_{11,+}(\xi)+\Phi_{12,+}(\xi) e^{n \varphi_{+}}\right) e^{2 n g_{+}(\xi)}\left(I+O\left(n^{-1}\right),\right. \\
& \xi=x, \quad z=x \sqrt{n} .
\end{aligned}
$$

Taking into consideration that $\Phi_{11,+}=\overline{\Phi_{12,+}}$, by formula 33 and $g_{+}(\xi)=i \pi \int_{x}^{\sqrt{2}} \rho(s) d s$ we obtain

$$
\begin{aligned}
H_{n, n}(x \sqrt{n}) \approx & \frac{1}{2}\left[\left(\frac{x-\sqrt{2}}{x+\sqrt{2}}\right)^{\frac{1}{4}} \cos \left(n \pi \int_{x}^{\sqrt{2}} \rho(s) d s+\frac{\pi}{4}\right)\right. \\
& \left.+\left(\frac{x+\sqrt{2}}{x-\sqrt{2}}\right)^{\frac{1}{4}} \cos \left(n \pi \int_{x}^{\sqrt{2}} \rho(s) d s-\frac{\pi}{4}\right)\right] \exp \left\{n \int_{-\sqrt{2}}^{\sqrt{2}} \ln |x-s| \rho(s) d s\right\} .
\end{aligned}
$$

It follows from asymptotics (37) and (38) that the zeroes of polynomials $H_{n, n}(z)$ are located in the domain $|z|<\sqrt{2 n}$. Their location is determined by vanishing of the square brackets in formula (38):

$$
\tan \left(n \pi \int_{x}^{\sqrt{2}} \rho(s) d s+\frac{\pi}{4}\right)=-\left(\frac{x-\sqrt{2}}{x+\sqrt{2}}\right)^{\frac{1}{2}}
$$

Calculating the integral in the argument of tangent, by the expression for $\rho(\xi)$ (34) we obtain

$$
\pi \int_{x}^{\sqrt{2}} \rho(s) d s=\frac{3}{2} x \sqrt{2-x^{2}}+(x \sqrt{2}-1) \operatorname{Arctan} \frac{x}{\sqrt{2-x^{2}}} .
$$


Equalling real and imaginary parts in (39), we finally get:

$$
\begin{aligned}
& \operatorname{Re}\left(\frac{3}{2} x \sqrt{2-x^{2}}+(x \sqrt{2}-1) \arctan \frac{x}{\sqrt{2-x^{2}}}\right)=\frac{1}{n}\left(\operatorname{Re} \arctan \frac{x-\sqrt{2}}{x+\sqrt{2}}+\pi j\right), \\
& \operatorname{Im}\left(\frac{3}{2} x \sqrt{2-x^{2}}+(x \sqrt{2}-1) \arctan \frac{x}{\sqrt{2-x^{2}}}\right)=\frac{1}{n}\left(\operatorname{Im} \arctan \frac{x-\sqrt{2}}{x+\sqrt{2}}+\pi k\right),
\end{aligned}
$$

where $j, k \in \mathbb{Z}$.

FiguRE 3. Numerical example of calculating zeroes in the first quadrant $x=$ $z / \sqrt{n}$ by formulae $(41)$ as $n=30,21 \leqslant j, k \leqslant 30$

Remark 1. It is easy to check that formulae (37) and (38) are generalizations of PlancherelRotach asymptotics [14] mentioned in the Introduction. Indeed, for real $z$ and $m=0$ by (12) we have $H_{n, 0}(z)=H_{n}(z)$, where $H_{n}(z)$ are classical Hermite polynomials. A comparison of Plancherel-Rotach formulae with (37) was made in the monograph by P. Deift [5]. Calculation of asymptotics for the zeroes of polynomials $H_{m, n}(z)$ for arbitrary $m, n \rightarrow \infty$ and $m-n=O(1)$ can be likely done by a method similar to one presented above.

\section{BIBLIOGRAPHY}

1. M. Bertola, T. Bothner. Zeros of large degree Vorob'ev-Yablonski polynomials via a Hankel determinant identity // Int. Math. Res. Not. 2015:19, 9330-9399 (2015).

2. R. J. Buckingham and P. D. Miller. Large-degree asymptotics of rational Painleve-II functions: noncritical behaviour // Nonlinearity. 27:10, 2498-2578 (2014).

3. Y. Chen and M.V. Feigin. Painlevé IV and degenerate Gaussian unitary ensembles // J. Phys. A. Math. Gen. 39:40, 12381-12393 (2006).

4. P.A. Clarkson. Special Polynomials Associated with Rational Solutions of the Painlevé Equations and Applications to Soliton Equations // Comp. Meth. Funct. Theory, 6:2, 329-401 (2006).

5. P. Deift. Orthogonal polynomials and random matrices: A Riemann-Hilbert approach. Courant Lecture Notes. New York Univ., New York (1999).

6. P. Deift, T. Kriecherbauer, K. T.-R. McLaughlin, S. Venakides, X. Zhou. Uniform asymptotics for polynomials orthogonal with respect to varying exponential weights and applications to universality questions in random matrix theory // Comm. Pure Appl. Math. 52:11, 1335-1425 (1999).

7. P. Deift, T. Kriecherbauer, K. T.-R. McLaughlin, S. Venakides, X. Zhou. Strong asymptotics of orthogonal polynomials with respect to exponential weights // Comm. Pure Appl. Math. 52:12, 1491-1552 (1999).

8. A.S. Fokas, A.R. Its, A.A. Kapaev and V.Yu. Novokshenov. Painlevé Transcendents. The Riemann-Hilbert Approach. Math. Surveys and Monographs. 128. AMS, Providence, RI (2006). 
9. A. Fokas, A. Its and A. Kitaev. Discrete Painlevé equations and their appearance in quantum gravity // Comm. Math. Phys. 142:2, 313-344 (1991).

10. P.J.Forrester. Log-gases and random matrices. Princeton University Press, Princeton (2010).

11. A.A. Kapaev, E. Hubert. A note on the Lax pairs for Painlevé equations // J. Phys. A. Math. Gen. 32:46, 8145-8156 (1999).

12. A.P. Magnus. Painleve-type differential equations for the recurrence coefficients of semi-classical orthogonal polynomials // J. Comp. Appl. Math. 57:1-2, 215-237 (1995).

13. V.Yu. Novokshenov and A.A. Shchelkonogov. Double scaling limit in the Painlevé IV equation and asymptotics of the Okamoto polynomials // In "Advances in the Mathematical Sciences". AMS Transl. 233, 199-210 (2014).

14. M. Plancherel, W. Rotach. Sur les valeurs asymptotiques des polynomes d'Hermite $H_{n}(x)=$ $(-I)^{n} e^{\frac{x^{2}}{2}} \frac{d^{n}}{d x^{n}}\left(e^{-\frac{x^{2}}{2}}\right) / /$ Commentarii Math. Helvetici. 1:1, 227-254 (1929).

15. E.B. Saff, V. Totik. Logarithmic potentials with external fields. "Grundlehren der mathematischen Wissenschaften", 317. Springer, Berlin (1997).

16. G. Szegö. Orthogonal polynomials. AMS, New York (1959).

17. M. Noumi and Y. Yamada. Symmetries in the fourth Painlevé equation and Okamoto polynomials // Nagoya Math. J. 153, 53-86 (1999).

Viktor Yurievich Novokshenov,

Institute of Mathematics CC USC RAS,

Chernyshevsky str. 112,

450008, Ufa, Russia

E-mail: novik53@mail.ru

Alexei Alexandrovich Schelkonogov,

Ufa State Aviation Technical University,

K. Marx str. 12,

450000, Ufa, Russia

E-mail: alexey91-91@mail.ru 